\title{
Preparation and Crystal Structure of a Phenoxo-Bridged Dinuclear Copper(II) Complex with $N$-Salicylidene-2-hydroxy-5-bromobenzylamine
}

\author{
Yoshihisa Kakuta, Michihiro Kurushima, Daisuke Yoshioka, and Masahiro MikurIYA ${ }^{\dagger}$ \\ Department of Chemistry, School of Science and Technology, Kwansei Gakuin University, \\ 2-1 Gakuen, Sanda 669-1337, Japan
}

\begin{abstract}
The title compound, phenoxo-bridged dinuclear copper(II) complex with $N$-salicylidene-2-hydroxy-5-bromobenzylamine $\left(\mathrm{H}_{2} \mathrm{shbb}\right)\left[\mathrm{Cu}_{2}(\mathrm{shbb})_{2}(\mathrm{dmso})_{2}\right] \cdot 2 \mathrm{dmso}(\mathrm{dmso}=$ dimethyl sulfoxide), was synthesized and the crystal structure was determined by the single-crystal X-ray diffraction method at $90 \mathrm{~K}$. It crystallizes in the monoclinic space group $P 2_{1} / n$ with $a=16.994(3) \AA, b=8.3932(16) \AA, c=15.755(3) \AA, \beta=111.547(3)^{\circ}, V=2090.2(7) \AA^{3}, D_{\mathrm{x}}=1.665 \mathrm{~g} / \mathrm{cm}^{3}$, and $Z=2$. The $R 1$ and $w R 2$ values are 0.0505 and 0.0649 , respectively, for 4759 reflections. The molecule is a phenoxo-bridged dinuclear copper(II) complex having an elongated square-pyramidal coordination geometry with the $O_{3} N$ basal atoms from the shbb ${ }^{2-}$ ligands and the apical $O$ atom from the dmso ligand.
\end{abstract}

(Received March 4, 2010; Accepted May 10, 2010; Published on web June 10, 2010)

$\mathrm{N}$-Salicylidene-2-hydroxybenzylamine and its substituted derivatives are unique ligands having two phenolic-oxygen and one imino-nitrogen donor atoms, forming mononuclear manganese (IV $)^{1}$ and vanadium(IV) ${ }^{2}$ complexes and dinuclear vanadium(V), ${ }^{2}$ iron(III), ${ }^{3}$ and copper(II) ${ }^{4}$ complexes with a meridional $\mathrm{O}, \mathrm{N}, \mathrm{O}$-chelating. Recently, we found that one of these ligands forms a hydrogen-bonded dimer structure in the crystal. ${ }^{5}$ Concerning the dimer structure, we are interested in the dinuclear structure of phenoxo-bridged copper(II) complexes with these ligands. Although we reported on the crystal structure for one of these, the title compound, before (Fig. 1), the X-ray diffraction data were measured at room temperature and were not sufficient to get good-quality data, and the structural detail had not been described in the literature. ${ }^{4}$ We also reported preliminary magnetic susceptibility data, and interpreted the magnetic data by a dimer model based on the room-temperature structure. ${ }^{4}$ In order to understand the magnetic property of the complex exactly, it is desirable to

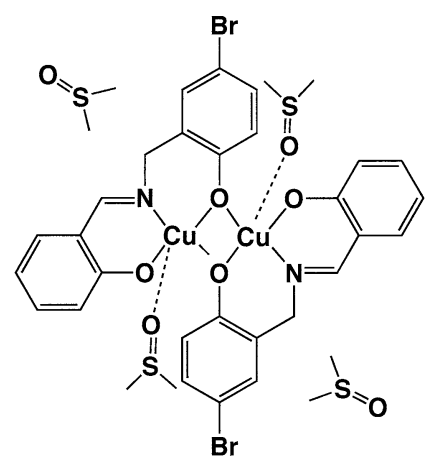

Fig. 1 Chemical structure of the title compound.

† To whom correspondence should be addressed. E-mail: junpei@kwansei.ac.jp confirm the low-temperature structure. In this study, we determined the crystal structure of the title compound at $90 \mathrm{~K}$.

The title compound was prepared by a previously reported procedure. $^{4}$ A mixture of 2-hydroxy-5-bromobenzylamine (20 $\mathrm{mg}, 0.1 \mathrm{mmol})$ and salicylaldehyde $(12 \mathrm{mg}, 0.1 \mathrm{mmol})$ dissolved in $3 \mathrm{~mL}$ of dimethyl sulfoxide was heated gently. To this solution was added copper(II) acetate $(18 \mathrm{mg}, 0.1 \mathrm{mmol})$, and the mixture was heated with stirring for $40 \mathrm{~min}$, and then filtered. The resulting green solution was allowed to stand at

Table 1 Crystal and experimental data

Chemical formula: $\mathrm{C}_{36} \mathrm{H}_{44} \mathrm{Br}_{2} \mathrm{Cu}_{2} \mathrm{~N}_{2} \mathrm{O}_{8} \mathrm{~S}_{4}$

Formula weight $=1047.87$

$T=90 \mathrm{~K}$

Crystal system: monoclinic

$a=16.994(3) \AA$

$b=8.3932(16) \AA$

$c=15.755(3) \AA$.

$V=2090.2(7) \AA^{3}$

$D_{\mathrm{x}}=1.665 \mathrm{~g} / \mathrm{cm}^{3}$

Radiation: $\operatorname{Mo} K_{\alpha}(\lambda=0.71073 \AA)$

$\mu\left(\right.$ Mo $\left.K_{\alpha}\right)=3.180 \mathrm{~mm}^{-1}$

Crystal size $=0.41 \times 0.25 \times 0.04 \mathrm{~mm}^{3}$

No. of reflections collected $=12136$

No. of independent reflections $=4759$

$\theta$ range for data collection: 1.51 to $28.41^{\circ}$

Data/Restraints/Parameters $=4759 / 0 / 244$

Goodness-of-fit on $F^{2}=0.949$

$R$ indices $[I>2 \sigma(I)]: R 1=0.0304, w R 2=0.0615$

$R$ indices (all data): $R 1=0.0505, w R 2=0.0653$

$(\Delta / \sigma)_{\max }=0.001$

$(\Delta \rho)_{\max }=0.846 \mathrm{e}^{-3}$

$(\Delta \rho)_{\min }=-0.374 \mathrm{e}^{-3}$

Measurement: Bruker Smart APEX CCD diffractometer

Program system: SHELXTL

Structure determination: Direct methods (SHELXS-97)

Refinement: full matrix least-squares (SHELXL-97)

CCDC deposition number: 765313 


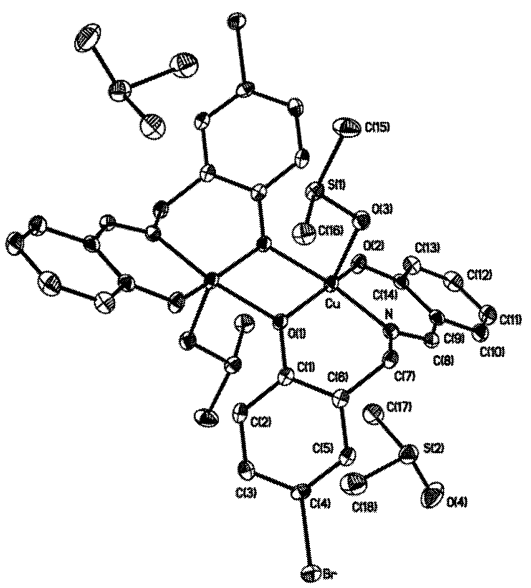

Fig. 2 ORTEP view of the title compound. The thermal ellipsoids are shown at the $50 \%$ probability level.

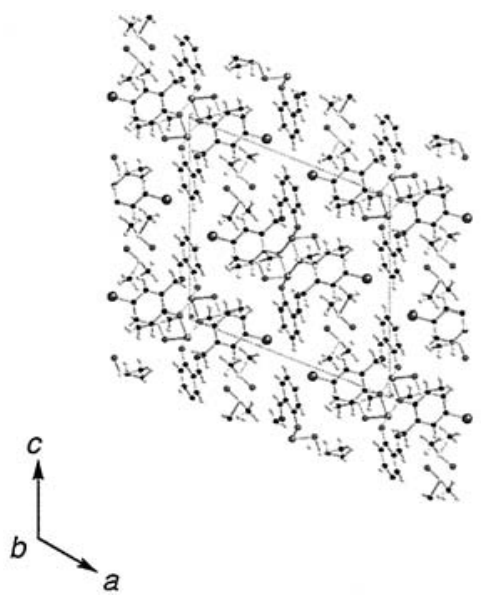

Fig. 3 Packing diagram of the title compound.

room temperature. After 2 days, deep-green crystals were deposited. These were collected by filtration, washed with methanol, and then dried under a vacuum over $\mathrm{P}_{2} \mathrm{O}_{5}$. Yield: 84 mg (73\%). Anal. Found: C, 41.10; H, 4.27; N, 2.66\%. Calcd for $\mathrm{C}_{32} \mathrm{H}_{32} \mathrm{Br}_{2} \mathrm{Cu}_{2} \mathrm{~N}_{2} \mathrm{O}_{6} \mathrm{~S}_{2} \cdot 2 \mathrm{C}_{2} \mathrm{H}_{6} \mathrm{SO}$ : C, 41.26; $\mathrm{H}, 4.23 ; \mathrm{N}, 2.67 \%$. IR(KBr): $v(\mathrm{ArH})$ 3057, 3044, 3003, 2989, 2858, $v(\mathrm{C}=\mathrm{N}) 1635$ $\mathrm{cm}^{-1}, \quad v(\mathrm{~S}=\mathrm{O}) 1024 \mathrm{~cm}^{-1}$. Diffuse reflectance spectrum: $\lambda_{\max }$ $281,385,644,730 \mathrm{sh}, 879 \mathrm{sh} \mathrm{nm}$. Magnetic moment: $\mu_{\text {eff }} / \mathrm{Cu}=$ $1.51 \mu_{\mathrm{B}}$ at $300 \mathrm{~K}$.

A preliminary examination was made, and data were collected on a Bruker CCD X-ray diffractometer (SMART APEX) using graphite-monochromated Mo- $K_{\alpha}$ radiation. Crystal data and details concerning data collection are given in Table 1 . The structures were solved by direct methods and refined by fullmatrix least-squares methods. The hydrogen atoms were inserted at their calculated positions and fixed there. All of the calculations were carried out on a Pentium IV Windows 2000 computer utilizing the SHELXTL software package.

The molecular structure drawn by ORTEP is shown in Fig. 2. Selected bond distances and angles are given in Table SI. The crystal structure consists of neutral centrosymmetric dinuclear entities and dmso molecules, showing the same crystal structure as that analyzed at room temperature. ${ }^{4}$ Two copper ions are bridged by phenolic-oxygen atoms of two Schiff-base ligands. The coordination geometry around the copper atom is an elongated square pyramid. One Schiff-base ligand is coordinated to the copper ion in a meridional fashion, giving a basal plane with a bridging phenolic-oxygen atom of the other Schiff-base ligand. The apical position is occupied by the dmso ligand. The basal plane, defined by $\mathrm{O} 1-\mathrm{N}-\mathrm{O} 2-\mathrm{O} 1 \mathrm{~A}$, deviates from the strict planar configuration within $\pm 0.17 \AA$, and the $\mathrm{Cu}$ atom is $0.20 \AA$ above the mean plane towards the oxygen atom of the dmso molecule. The bond distances $\mathrm{Cu}-\mathrm{O} 1$ [1.998(2) $\mathrm{A}]$,

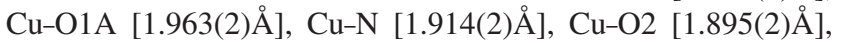
and $\mathrm{Cu}-\mathrm{O} 3[2.387(2) \AA]$ are comparable to those found at room temperature $[\mathrm{Cu}-\mathrm{O} 12.001(8) \AA, \quad \mathrm{Cu}-\mathrm{O} 1 \mathrm{~A} \quad 1.970(9) \AA, \quad \mathrm{Cu}-\mathrm{N}$

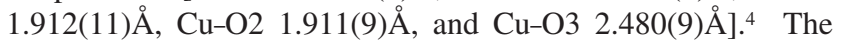
difference in the bond distances between at room temperature and $90 \mathrm{~K}$ may come from the crystal-packing effect. The $\mathrm{Cu} \cdots \mathrm{Cu}$ distance within the dinuclear unit is 3.0862(8) $\AA$. The $\mathrm{Cu}-\mathrm{O}$ (phenolic) $-\mathrm{Cu}$ bridging angle is $102.37(7)^{\circ}$. These important bonding parameters are essentially the same as those found at room temperature $[\mathrm{Cu} \cdots \mathrm{Cu} \quad 3.077(2) \AA, \quad \mathrm{Cu}-\mathrm{O}-\mathrm{Cu}$ $\left.101.6(4)^{\circ}\right]$. Therefore, we can suppose a dimer model without any significant structural change for the temperature dependence of the magnetic susceptibility. In the Schiff-base moiety, the NC7 distance is $1.475(3) \AA$, which is normal for a single bond, while the $\mathrm{N}-\mathrm{C} 8$ bond length of 1.282(3) $\AA$ shows a double-bond character. The $\mathrm{C} 7, \mathrm{~N}, \mathrm{C} 8$, and $\mathrm{C} 9$ moiety is essentially flat (r. m. s. deviation from the mean plane is $0.0008 \AA$ ), which is consistent with the $\mathrm{sp}^{2}$ hybrid character of the $\mathrm{N}$ atom. The torsion angles around $\mathrm{C} 7, \mathrm{C} 6-\mathrm{C} 7-\mathrm{N}-\mathrm{C} 8, \mathrm{C} 1-\mathrm{C} 6-\mathrm{C} 7-\mathrm{N}$, and $\mathrm{C} 5-$ C6-C7-N (Table SI) show that this molecule twists slightly at the $\mathrm{C} 7$ atom, giving a dihedral angle of the aromatic rings containing $\mathrm{C} 1$ and $\mathrm{C} 9$ of $57.30(9)^{\circ}$. This structural feature is also found in the ligand, itself, ${ }^{5}$ and may be in harmony with the coordination to metal ion in a meridional fashion of this ligand. In the crystal, each molecule is well separated, giving a good example of the dimer model. The closest contact between the dinuclear unit and the crystal dmso is 3.300(3) $\mathrm{A}$ of O4...C7 ($x+1 / 2, y-1 / 2,3 / 2-z$ ) (Fig. 2). Further work on the magnetic properties is in progress in our laboratory.

\section{Acknowledgements}

The present work was partially supported by Grants-in-Aid for Scientific Research Nos. 19550074 and 22550066 from the Ministry of Education, Culture, Sports, Science and Technology.

\section{References}

1. M. Mikuriya, D. Jie, Y. Kakuta, and T. Tokii, Bull. Chem. Soc. Jpn., 1993, 66, 1132.

2. J. Dai, S. Akiyama, M. Munakata, and M. Mikuriya, Polyhedron, 1994, 13, 2495.

3. M. Mikuriya, Y. Kakuta, R. Nukada, T. Kotera, and T. Tokii, Bull. Chem. Soc. Jpn., 2001, 74, 1425.

4. M. Mikuriya, Y. Kakuta, K. Kawano, and T. Tokii, Chem. Lett., 1991, 2031.

5. Y. Kakuta, C. Myojo, D. Yoshioka, Z. Zhu, and M. Mikuriya, Anal. Sci., 2008, 24, x267. 